\title{
ON VECTOR-VALUED SPECTRA
}

\author{
ROBIN HARTE \\ Trinity College, Dublin, Ireland \\ e-mail:rharte@maths.tcd.ie \\ and CIARAN TAYLOR* \\ Institute of Technology, Tallaght, Ireland \\ e-mail:ciaran.taylor@it-tallaght.ie
}

(Received 27 July, 1998; revised 17 May, 1999)

\begin{abstract}
Elements $\alpha \in A \otimes E$ of the tensor product of a Banach algebra $A$ and a Banach space $E$ induce systems $\left\{\psi(\alpha): \psi \in E^{*}\right\}$ of elements of $A$ indexed by the dual space $E^{*}$, whose joint spectrum belongs to the second dual $E^{* *}$. In this note we investigate when the spectrum actually lies in $E \subseteq E^{* *}$, and extend the spectral mapping theorem $P \sigma_{A}(\alpha)=\sigma_{A} P(\alpha)$ to polynomial mappings $P: E \rightarrow F$ between Banach spaces. When the algebra $A$ is commutative and the Banach space $E=B$ is another algebra we also reach a sort of vector-valued Gelfand theory.
\end{abstract}

1991 Mathematics Subject Classification. 47A13.

If $A$ is a complex Banach algebra, with identity 1 , and $\left(a_{x}\right)_{x \in X}$ a family of elements in $A$, then the left and the right spectrum of $a \in A^{X}$ are defined as subsets of the corresponding families of complex numbers ([2];[3, Definition 11.4.1]):

$$
\sigma_{A}^{l e f t}(a)=\left\{\lambda \in \mathbf{C}^{X}: 1 \notin \sum_{x \in X} A\left(a_{x}-\lambda_{x}\right)\right\}
$$

and

$$
\sigma_{A}^{r i g h t}(a)=\left\{\lambda \in \mathbf{C}^{X}: 1 \notin \sum_{x \in X}\left(a_{x}-\lambda_{x}\right) A\right\} .
$$

Thus if, for example, $\lambda \in \mathbf{C}^{X}$ is not in the left spectrum of $a \in A^{X}$ there is $b=\left(b_{x}\right)_{x \in X}$ in $A$, vanishing for all but finitely many $x \in X$, for which $\sum_{x \in X} b_{x}\left(a_{x}-\lambda_{x}\right)=1$. The right and left spectra are in a sense the same, being interchanged by "reversal of products". We recall that they are compact subsets of $\mathbf{C}^{X}$, in the topology of pointwise convergence on $X$, possibly empty, and ([3, Theorem 11.4.2]) subject to the oneway spectral mapping theorem for 'polynomials':

$$
p \sigma_{A}^{\text {left }}(a) \subseteq \sigma_{A}^{\text {left }} p(a), p \sigma_{A}^{r i g h t}(a) \subseteq \sigma_{A}^{r i g h t} p(a),
$$

where a polynomial $p=\left(p_{y}\right)_{y \in Y}: A^{X} \rightarrow A^{Y}$ is a system of members of the free algebra generated by the coordinates $z_{x}$ and the identity 1 . If in particular the system $a=\left(a_{x}\right)_{x \in X}$ is commutative then by [3, Theorem 11.4.4] the spectra $\sigma_{A}^{\text {left }}(a), \sigma_{A}^{\text {right }}(a)$ are nonempty, and there is equality in $(0.3)$.

\footnotetext{
*The second author acknowledges partial support from an Enterprise Ireland basic research scholarship.
} 
If, for example, $X=\{1,2, \ldots, n\}$ then these add up to the "so-called Harte spectrum" [4] of the $n$-tuple $a=\left(a_{1}, a_{2}, \ldots, a_{n}\right)$; more generally $\lambda \in \mathbf{C}^{X}$ is in $\sigma_{A}^{\text {left }}(a)$ if and only if every finite restriction of $\lambda$ is in the left spectrum of the corresponding restriction of $a \in A^{X}$. Infinite indexing systems $X$ have however the possibility of nontrivial structure: thus ([2, Lemma 1], [3, Theorem 11.4.3]) we have the following result.

Lemma 1. If $\lambda \in \mathbf{C}^{X}$ is in the left or the right spectrum of $a \in A^{X}$, and if $a: X \rightarrow A$ is either bounded, or continuous, or homomorphic, or linear, then so is $\lambda: X \rightarrow \mathbf{C}$.

Proof. This is the one way spectral mapping theorem (0.3), together with the spectral theory of a single element: for arbitrary $x, x^{\prime}, x^{\prime \prime}$ in $X$ we find that $\lambda_{x}$, $\lambda_{x^{\prime}}-\lambda_{x}$ and $p\left(\lambda_{x}, \lambda_{x^{\prime}}, \lambda_{x^{\prime \prime}}\right)$ are in $\sigma\left(a_{x}\right), \sigma\left(a_{x^{\prime}}-a_{x}\right)$ and $\sigma p\left(a_{x}, a_{x^{\prime}}, a_{x^{\prime \prime}}\right)$, respectively, and hence we have

$$
\left|\lambda_{x}\right| \leq\left\|a_{x}\right\| ;\left|\lambda_{x^{\prime}}-\lambda_{x}\right| \leq\left\|a_{x^{\prime}}-a_{x}\right\| ;\left|p\left(\lambda_{x}, \lambda_{x^{\prime}}, \lambda_{x^{\prime \prime}}\right)\right| \leq\left\|p\left(a_{x}, a_{x^{\prime}}, a_{x^{\prime \prime}}\right)\right\| .
$$

The first of these inequalities transmits boundedness, the second continuity, and the third ensures that $\left(\lambda_{x}, \lambda_{x^{\prime}}, \lambda_{x^{\prime \prime}}\right)$ is subjected to any polynomial identity satisfied by $\left(a_{x}, a_{x^{\prime}}, a_{x^{\prime \prime}}\right)$.

Thus, for example, if $X=\mathbf{N}$ and $a=\left(a_{n}\right) \in \ell_{1}(A)$ then

$$
\lambda \in \sigma_{A}^{\text {left }}(a) \subseteq \mathbf{C}^{\mathbf{N}} \Longrightarrow \lambda \in \ell_{1} ;
$$

if instead $X=\Omega$ is a topological space and $a \in C(\Omega, A)$, then

$$
\lambda \in \sigma_{A}^{\text {left }}(a) \subseteq \mathbf{C}^{\Omega} \Longrightarrow \lambda \in C(\Omega)
$$

if, in particular, $X=F$ is a Banach space and $a \in B L(F, A)$ then

$$
\lambda \in \sigma_{A}^{\text {left }}(a) \subseteq \mathbf{C}^{F} \Longrightarrow \lambda \in F^{*} .
$$

In the ultimate special case ([2, Theorem 1]; [3, Theorem 11.4.3]) if $a_{x}=x$ for each $x \in X=A$, then the left and the right spectrum of $a$ reduce to the Gelfand 'maximal ideal space' of multiplicative linear functionals:

$$
\sigma_{A}^{\text {left }}(a)=\sigma_{A}^{\text {right }}(a)=\sigma(A) .
$$

In this note we would like to focus on the situation in which

$$
\alpha=\sum_{n=1}^{\infty} a_{n} \otimes e_{n} \in A \otimes E
$$

belongs to a uniform cross-normed tensor product [3, Definition 11.7.1] of $A$ with a Banach space $E$; examples include operator matrices and continuous vector-valued functions. Waelbroeck [5],[6] has looked here for a functional calculus involving holomorphic functions in infinitely many variables. Evidently $\alpha$ induces a bounded linear operator $\alpha_{\wedge}: \psi \mapsto \psi(\alpha)$ from the dual space $E^{*}$ into $A$, where we write 


$$
\alpha_{\wedge}(\psi)=\psi(\alpha)=\sum_{n=1}^{\infty} \psi\left(e_{n}\right) a_{n} \in A
$$

if $\psi \in E^{*}$ and $\alpha \in A \otimes E$ is given by (1.6). The spectrum of $\alpha_{\wedge} \in B L(F, A)$, with $F=E^{*}$, lies by (1.4) in the second dual $E^{* *}$ of the space $E$, and would be a candidate for the spectrum of the system $\alpha \in A \otimes E$; we would however prefer a spectrum lying in the space $E$, writing more intuitively

$$
\begin{aligned}
& \sigma_{A}^{l e f t}(\alpha)=\left\{x \in E: 1 \notin \sum_{\psi \in E^{*}} A(\psi(\alpha)-\psi(x))\right\}, \\
& \sigma_{A}^{r i g h t}(\alpha)=\left\{x \in E: 1 \notin \sum_{\psi \in E^{*}}(\psi(\alpha)-\psi(x)) A\right\} .
\end{aligned}
$$

Thus the spectrum of $\alpha$ is essentially the intersection of the space $E \cong E^{\wedge} \subseteq E^{* *}$ with the spectrum of $\alpha_{\wedge}$. The good news is that, provided we stay in the 'projective' product, the spectra of $\alpha$ and $\alpha_{\wedge}$ coincide. then

Theorem 2. If $\alpha=\sum_{n=1}^{\infty} a_{n} \otimes e_{n} \in A \otimes E$ with $\max _{n}\left\|a_{n}\right\|<\infty$ and $\sum_{n}\left\|e_{n}\right\|<\infty$,

$$
\sigma_{A}^{l e f t}\left(\alpha_{\wedge}\right)=\sigma_{A}^{l e f t}(\alpha)^{\wedge} \subseteq E^{\wedge} \subseteq E^{* *}, \sigma_{A}^{r i g h t}\left(\alpha_{\wedge}\right)=\sigma_{A}^{r i g h t}(\alpha)^{\wedge} \subseteq E^{\wedge} \subseteq E^{* *} .
$$

Proof. If we write $e^{\wedge}: E^{*} \rightarrow \ell_{1}$ for the operator which sends $\psi \in E^{*}$ to $\left(\psi\left(e_{n}\right)\right) \in \ell_{1}$, then each $\xi \in \sigma_{A}^{\text {left }}(\alpha)$ is 'majorized' [3, Definition 10.1.1] by $e^{\wedge}$ :

$$
|\xi(\psi)| \leq\|a\|_{\infty}\left|e^{\wedge}(\psi)\right|
$$

and hence by [1, Lemma 1] (cf. [3, Theorem 5.5.3]) factors through $e^{\wedge}$ :

$$
\xi=\theta \circ e^{\wedge} \text { with } \theta \in \ell_{1}^{*}
$$

obtained by Hahn-Banach extension from the functional $\theta_{0}: e^{\wedge}\left(E^{*}\right) \rightarrow \mathbf{C}$, defined by setting $\theta_{0}\left(e^{\wedge}(\psi)\right)=\xi(\psi)$. Thus there is $\lambda \in \ell_{\infty}$ for which

$$
\xi=\sum_{n=1}^{\infty} \lambda_{n} e_{n}^{\wedge} \in E^{\wedge} .
$$

When the element $\alpha \in A \otimes E$ is commutative, in the sense that $\left\{\psi(\alpha): \psi \in E^{*}\right\} \subseteq A$ is commutative, this extends to all uniform products.

Theorem 3. If $\alpha \in A \otimes E$ is commutative, then (2.1) holds. If, in particular, $A$ is commutative then

$$
\sigma_{A}^{\text {left }}\left(\alpha_{\wedge}\right)=\sigma_{A}^{r i g h t}\left(\alpha_{\wedge}\right)=\{\varphi(\alpha): \varphi \in \sigma(A)\}^{\wedge},
$$

where $\sigma(A) \subseteq A^{*}$ is the 'maximal ideal space' of $A$ and where if $\alpha \in A \otimes E$ and $\varphi \in \sigma(A)$ we have 


$$
\varphi(\alpha)=\lim \left\{\sum_{j \in J} \varphi\left(a_{j}\right) e_{j}: \sum_{j \in J} a_{j} \otimes e_{j} \rightarrow \alpha\right\} \in E .
$$

The Gelfand mapping

$$
\alpha \mapsto \alpha^{\wedge}: A \otimes E \rightarrow C(\sigma(A), E)
$$

is continuous, and the spectrum of $\alpha \in A \otimes E$ is the range of the Gelfand transform $\alpha^{\wedge}: \varphi \mapsto \varphi(\alpha)$.

Proof. Suppose first that the algebra $A$ is commutative: if $\alpha \in A \otimes E$ and if $\xi \in E^{* *}$ is in $\sigma_{A}^{\text {left }}\left(\alpha_{\wedge}\right)$ then

$$
1 \notin \sum_{\psi \in E^{*}} A(\psi(\alpha)-\xi(\psi))
$$

By Gelfand theory [3, Theorem 9.6.3], there is $\varphi \in \sigma(A)$ for which

$$
\left\{\psi(\alpha)-\xi(\psi): \psi \in E^{*}\right\} \subseteq \varphi^{-1}(0),
$$

which means, if $\alpha \in A \otimes E$, that

$$
\lim \left\{\sum_{j \in J} \varphi\left(a_{j}\right) \psi\left(e_{j}\right): \sum_{j \in J} a_{j} \otimes e_{j} \rightarrow \alpha\right\}=\xi(\psi) \text { for each } \psi \in E^{*} .
$$

But this means that

$$
\xi=\lim \left\{\left(\sum_{j \in J} \varphi\left(a_{j}\right) e_{j}\right)^{\wedge}: \sum_{j \in J} a_{j} \otimes e_{j} \rightarrow \alpha\right\} \in E^{\wedge} \subseteq E^{* *} .
$$

For the continuity observe that, with $\alpha \in A \otimes E$ and arbitrary $\varphi \in \sigma(A)$, we have

$$
|\varphi(\alpha)| \leq \sup _{\|\psi\| \leq 1}|\psi(\alpha)| .
$$

If more generally $\alpha \in A \otimes E$ is commutative, then this argument applies to the closed (unital) subalgebra $B \subseteq A$ generated by the elements $\left\{\psi(\alpha): \psi \in E^{*}\right\}$, so that

$$
\sigma_{A}^{l e f t}(\alpha) \subseteq \sigma_{B}^{l e f t}(\alpha) \subseteq E^{\wedge}
$$

Theorem 3 survives if the algebra $A$ is commutative modulo its radical, or if $\alpha \in A \otimes E$ is for example 'quasi-commutative' [3, Definition 11.8.3], in the sense that all the commutators $\psi(\alpha) \theta(\alpha)-\theta(\alpha) \psi(\alpha)$ commute with each $\phi(\alpha)$. Theorem 2 sometimes holds for non commutative $A$, nonreflexive $E$ and arbitrary products.

EXAMPLE 4. If $E=c_{0}$, so $E^{* *} \cong \ell_{\infty}$, and if

$$
\alpha \in A \otimes c_{0} \subseteq A \odot c_{0} \cong c_{0}(A) \subseteq \ell_{\infty}(A)
$$


then there is implication

$$
y \in \sigma_{A}^{l e f t}(\alpha) \cup \sigma_{A}^{r i g h t}(\alpha) \subseteq \ell_{\infty} \Longrightarrow y \in c_{0} .
$$

Proof. This result follows from Lemma 1.

The 'polynomials' of (0.3), specialised to the systems $\alpha_{\wedge}=(\psi(\alpha))_{\psi \in E^{*}}$, are generated by "co-ordinates" $\left(z_{\psi}\right)_{\psi \in E^{*}}$, and continue to act on the " $E$-valued" spectra (1.8) and (1.9). In this context however there are more serious polynomials $P: E \rightarrow F$, induced by symmetric bounded multilinear operators:

$$
P=\sum_{n=0}^{N} P_{n} \in P \operatorname{Poly}(E, F) \text { with } P_{n}(x)=P_{n}^{\vee}(x, x, \ldots, x) \in \operatorname{Poly}_{n}(E, F)(x),
$$

where $P_{n}^{\vee}: E^{n} \rightarrow F$ is bounded symmetric $n$-linear. Thus $\operatorname{Poly}_{1}(E, F)=B L(E, F)$ is just the space of bounded linear operators and $\operatorname{Poly}_{0}(E, F)=F$ is the constants; a product $\psi \cdot T: x \mapsto \psi(x) T(x)$ with $\psi \in E^{*}$ and $T \in B L(E, F)$ is a rather special kind of element of $\operatorname{Poly}_{2}(E, F)$. These 'polynomials' also act on the (projective) product $A \otimes E$ if we define (cf [5, Chapter VIII p. 127], [6, p. 106])

$$
P(\alpha)=\sum_{n=0}^{N} \sum_{|k|=n} a_{k} \otimes P_{n}^{\vee}\left(e_{k}\right) \text { if } \alpha=\sum_{m=0}^{\infty} a_{m} \otimes e_{m},
$$

where we write

$$
e_{k}=\left(e_{k_{1}}, e_{k_{2}}, \ldots, e_{k_{n}}\right) \text { and } a_{k}=a_{k_{1}} a_{k_{2}} \ldots a_{k_{n}} \text { if } k=k_{1} k_{2} \ldots k_{n} .
$$

It has to be checked that $P(\alpha)$ is well-defined; then the spectral mapping theorem holds.

Theorem 5. If $\alpha \in A \otimes E$ is arbitrary and if $P: E \rightarrow F$ is a polynomial, there is inclusion

$$
P \sigma_{A}^{l e f t}(\alpha) \subseteq \sigma_{A}^{l e f t} P(\alpha) \text { and } P \sigma_{A}^{r i g h t}(\alpha) \subseteq \sigma_{A}^{r i g h t} P(\alpha)
$$

with equality if $\alpha$ is commutative.

Proof. We claim that, acting on $A \otimes E$, a weak remainder theorem (cf [3, Theorem 11.2.1]) is valid for polynomials $P \in \operatorname{Poly}(E, F)$ : for arbitary $\theta \in F^{*}$ we have

$$
\theta(P(\alpha)-P(x)) \in \mathrm{cl} \sum_{\psi \in E^{*}} A(\psi(\alpha)-\psi(x))
$$

It is clear that (5.2) holds if $P$ is either a constant or a linear operator, and holds if $P=Q+R$ where $Q$ and $R$ are polynomials for which (5.2) holds. It is therefore sufficient to establish (5.2) for $P \in \operatorname{Poly}_{n}(E, F)$ for each $n \in \mathbf{N}$. Generally if $P \in \operatorname{Poly}_{n+1}(E, F)$, if $\alpha=\sum_{m=1}^{\infty} a_{m} \otimes e_{m} \in A \otimes E$ and $x \in E$, we have 


$$
P(\alpha)-P(x)=\sum_{|k|+|j|=n}\left(a_{k} \otimes 1\right)\left(\sum_{m=1}^{\infty} a_{m} \otimes P^{\vee}\left(e_{k}, x^{j}, e_{m}\right)-1 \otimes P^{\vee}\left(e_{k}, x^{j}, x\right)\right) .
$$

This remainder theorem gives the one way inclusion (5.1); if, in particular, $\alpha$ is commutative then by [3, Theorem 11.4.4] together with (2.1)

$$
y \in \sigma_{A}^{l e f t} P(\alpha) \subseteq F \Longrightarrow \exists x \in E \text { with }(x, y) \in \sigma_{A}^{l e f t}(\alpha, P(\alpha))
$$

and by [3, Theorem 11.2.6]

$$
(x, y) \in \sigma_{A}^{l e f t}(\alpha, P(\alpha)) \Longrightarrow y=P(x) .
$$

To prove (5.5) we write $Q(z, w)=w-P(z)$ and notice that

$$
Q(x, y) \in Q \sigma_{A}^{\text {left }}(\alpha, P(\alpha)) \subseteq \sigma_{A}^{\text {left }} Q(\alpha, P(\alpha))=\sigma_{A}^{\text {left }}(0)=\{0\} .
$$

When $A$ is commutative and we stay in the projective product, Waelbroeck [6] establishes a functional calculus $f \mapsto f(\alpha)$ from functions 'holomorphic' near $\sigma_{A}(\alpha) \subseteq E$ to $A$. If, in particular, $E=B$ is another Banach algebra and the crossnorm on $A \otimes E$ is such that $A \otimes E=A \otimes B$ is again a Banach algebra, then there are three kinds of 'spectrum' induced on $\alpha \in A \otimes B$ :

$$
\sigma_{A}(\alpha) \subseteq B ; \sigma_{B}(\alpha) \subseteq A ; \sigma_{A \otimes B}(\alpha) \subseteq \mathbf{C}
$$

These are sometimes related. See [2, (2.4)].

Theorem 6. If $A$ and $B$ are Banach algebras with $A$ commutative, then for arbi$\operatorname{trary} \alpha \in A \otimes B$

$$
\sigma_{A \otimes B}^{\text {left }}(\alpha)=\bigcup\left\{\sigma_{B}^{\text {left }}(b): b \in \sigma_{A}^{\text {left }}(\alpha)\right\}, \sigma_{A \otimes B}^{r i g h t}(\alpha)=\bigcup\left\{\sigma_{B}^{\text {right }}(b): b \in \sigma_{A}^{\text {right }}(\alpha)\right\} .
$$

Proof. Recall [3, Theorem 11.7.5] that if $a \in A^{X}$ and $b \in B^{Y}$ are arbitrary there is equality

$$
\sigma_{A \otimes B}^{\text {left }}(a \otimes 1,1 \otimes b)=\sigma_{A}^{\text {left }}(a) \times \sigma_{B}^{\text {left }}(b):
$$

inclusion one way is obvious, since if for example $\sum_{x \in X} a_{x}^{\prime}\left(a_{x}-\lambda_{x}\right)=1$ then

$$
\sum_{x \in X}\left(a_{x}^{\prime} \otimes 1\right)\left(\left(a_{x}-\lambda_{x}\right) \otimes 1\right)+\sum_{y \in Y}\left(1 \otimes b_{y}^{\prime}\right)\left(1 \otimes\left(b_{y}-\mu_{y}\right)\right)=1 \otimes 1
$$

with $b_{y}^{\prime}=0$. Conversely if $(\lambda, \mu)$ is in the right hand side, so that $M=\operatorname{cl} \sum_{x \in X} A\left(a_{x}-\lambda_{x}\right)$ and $N=\operatorname{cl} \sum_{y \in Y} B\left(b_{y}-\mu_{y}\right)$ are proper closed left ideals of $A$ and $B$, then by the Hahn-Banach theorem there are $\varphi \in A^{*}$ and $\theta \in B^{*}$ for which $\varphi(1)=1=\theta(1)$ with $\varphi(M)=\{0\}=\theta(N)$. But now since the product $A \otimes B$ is uniform, the linear functional $\varphi \otimes \theta$ is well-defined and bounded on $A \otimes B$, and satisfies

$(\varphi \otimes \theta)(1 \otimes 1)=1$ with $(\varphi \otimes \theta)(M \otimes B+A \otimes N)=\{0\}$. 
Towards (6.1) suppose $b \in \sigma_{A}^{\text {left }}(\alpha)$, so that by Theorem 3 there is $\varphi \in \sigma(A)$ for which $b=\varphi(\alpha)$. By (6.2) it follows from $\mu \in \sigma_{A}^{\text {left }} \varphi(\alpha)$ that

$$
(\varphi, \mu) \in \sigma_{A \otimes B}^{l e f t}(A \otimes 1,1 \otimes \varphi(\alpha)) \subseteq A^{*} \times \mathbf{C},
$$

or equivalently

$$
(\varphi, \mu) \in \sigma_{A \otimes B}^{l e f t}(A \otimes 1, \alpha)
$$

since by [3, Theorem 11.3.5] $\alpha-1 \otimes \varphi(\alpha)$ is in the closed left ideal of $A \otimes B$ generated by

$$
A \otimes 1-\varphi \otimes 1=\{(c-\varphi(c)) \otimes 1: c \in A\}
$$

By the one way spectral mapping theorem (0.3) this gives $\mu \in \sigma_{A \otimes B}^{\text {left }}(\alpha)$. Conversely this implies by the two way spectral mapping theorem that there is $\varphi \in \sigma(A)$ for which (6.4) holds; hence we have established (6.3), which by (6.2) gives $\mu \in \sigma_{B}^{\text {left }} \varphi(\alpha)$, with of course $b=\varphi(\alpha) \in \sigma_{A}^{\text {left }}(\alpha)$.

From Theorem 6 we can deduce that the spectrum of a commutative operator matrix, an upper triangular operator matrix, or a continuous vector-valued function, is what it ought to be [3, Theorem 11.7.7; (11.7.7.13), (11.7.7.16)], and also give an alternative proof of Allen's theorem [3, Theorem 11.7.9] about holomorphic one sided inverses. Specifically Theorem 6 offers a sort of vector-valued Gelfand theorem for $A \otimes B$ : if $A$ is commutative then $\alpha$ is left, or right, invertible in $A \otimes B$ if and only if $\varphi(\alpha)$ is left, or right, invertible in $B$ for every $\varphi \in \sigma(A)$.

\section{REFERENCES}

1. M. R. Embry, Factorization of operators on Banach spaces, Proc. Amer. Math. Soc. 38 (1973), 587-590.

2. R. E. Harte, The spectral mapping theorem in many variables in Proc. Seminar 'Uniform algebras', University of Aberdeen 1973, 59-63; MR 50:\#14226.

3. R. E. Harte, Invertibility and singularity (Dekker, New York, 1988); MR 89d:47001.

4. F.-H. Vasilescu, Math. Reviews. MR 95d:47003.

5. L. Waelbroeck, Topological vector spaces and algebras, Lecture Notes in Mathematics \#230 (Springer-Verlag, 1971).

6. L. Waelbroeck, The holomorphic functional calculus and infinite dimensional holomorphy in Proc. on infinite dimensional holomorphy 1973, 101-108 Lecture Notes in Mathematics \#364 (Springer-Verlag, 1974). 